\title{
Combining Hebbian and reinforcement learning in a minibrain model
}

\author{
R. J. C. Bosman, W. A. van Leeuwen and B. Wemmenhove \\ Institute for Theoretical Physics, University of Amsterdam, Valckenierstraat 65, \\ 1018 XE Amsterdam, The Netherlands
}

\begin{abstract}
A toy model of a neural network in which both Hebbian learning and reinforcement learning occur is studied. The problem of 'path interference', which makes that the neural net quickly forgets previously learned input-output relations is tackled by adding a Hebbian term (proportional to the learning rate $\eta$ ) to the reinforcement term (proportional to $\rho$ ) in the learning rule. It is shown that the number of learning steps is reduced considerably if $1 / 4<\eta / \rho<1 / 2$, i.e., if the Hebbian term is neither too small nor too large compared to the reinforcement term.
\end{abstract}




\section{Introduction}

The central question which we address in this article is in what way a biological neural network, i.e., the brain, or, more generally, a part of the nervous system of an animal or human being, may learn to realize input-output relations. By biological we here mean: realizable with the help of elements occurring in nature, e.g., neurons or chemical substances that may influence other neurons or the synaptic efficacy.

An example of an input-output relation is a motor task, like catching a prey, in reaction to visual, auditive, or other input. Many attempts to explain the way input-output relations of this kind might be realized by (artificial) neural nets are encountered in the literature, most of which are not satisfactory from a biological point of view as we will illustrate in subsection 1.1.

It is the purpose of this article to combine ideas which do satisfy certain biological constraints and study a toy model, in particular with respect to its ability to learn and realize input-output relations.

The widely accepted idea of Hebbian learning [5] at the one hand will be combined with some rule that implements a feedback signal at the other hand, in a way that, in principle, might be biologically realizable. Without the addition of any feedback-signal, learning of prescribed input-output relations - whether in reality or in a model - is, of course, impossible.

\subsection{Artificial learning rules}

If one wants a network to learn to realize input-output relations, there are various well-known prescriptions, associated with names like perceptron learning rule, back-propagation or Boltzmann machines [12,7]. None of these, however, model the functioning of real brains, since the learning rules in question violate the existing biological limitations. In order to illustrate this statement, let us give an example.

Consider a single layered feed-forward network, i.e., a network consisting of an input and an output layer only, in which signals are sent by neurons of the input layer to neurons of the output layer, and not the other way around. Let $w_{i j}$ be the strengths or 'weights' of the connections in this simple net. In 1962, Rosenblatt [13] proved that such a network will realize desired input-output relations if, a finite number of times, the weights are adapted according to the rule

$$
w_{i j} \rightarrow w_{i j}+\Delta w_{i j}
$$


with

$$
\Delta w_{i j}=\varepsilon\left(x_{\mathrm{T} i}-x_{\mathrm{O} i}\right) x_{j}
$$

where $x_{\mathrm{T} i}$ is the desired or target output of neuron $i$, and $x_{\mathrm{O} i}$ is its actual output. Furthermore, $x_{j}$ is the state of the pre-synaptic input neuron $j$ and $\varepsilon$ is some function of the neuron states and properties of neurons $i$ and $j$. This learning rule can not be realized by a biological neural net since neuron $i$, producing $x_{\mathrm{O} i}$, cannot know that it should produce $x_{\mathrm{T} i}$. If, e.g., an animal does not succeed in catching a prey, its neurons get no specific feedback individually, on what the right output $x_{\mathrm{T} i}$ should have been. Hence, $x_{\mathrm{T} i}-x_{\mathrm{O} i}$ cannot be determined by the biological system, and, therefore, neither can it adapt the weights according to (2). Consequently, the perceptron learning rule (2) is unsuitable for a realistic modeling of the way in which a biological neural net can learn and realize input-output relations. Similar observations can be made for back-propagation or Boltzmann machines.

\subsection{Biological learning rules; Hebbian learning and reinforcement learning}

Already in 1949, Hebb suggested [5] that, in biological systems, learning takes place through the adaptation of the strengths of the synaptic interactions between neurons, depending on the activities of the neurons involved. In a model using binary neurons, i.e., $x_{i}=0$ or $x_{i}=1$, the most general form of a learning rule based on this principle is a linear function in $x_{i}$ and $x_{j}$ since $x_{i}^{2}=x_{i}$ and $x_{j}^{2}=x_{j}$. It therefore reads

$$
\Delta w_{i j}=a_{i j}+b_{i j} x_{i}+c_{i j} x_{j}+d_{i j} x_{i} x_{j}
$$

In a biological setting, the coefficients $a_{i j}, b_{i j}, c_{i j}$ and $d_{i j}$ in this learning rule can only depend on locally available information, such as the values of the membrane potential

$$
h_{i}=\sum_{j} w_{i j} x_{j}
$$

and the threshold potential $\theta_{i}$ of neuron $i$. In this way, the system adapts its weights without making use of neuron-specific information, like, e.g., $x_{\mathrm{T} i}$, of which there can be no knowledge, locally, at the position of the synapse.

In a recurrent neural net, a Hebbian learning rule suffices to store patterns $[6,12]$ if all neurons are clamped to the patterns which are to be learned during a certain period, the 'learning stage'. In feed-forward networks, however, only 
the neurons of the input layers are clamped, and some kind of feed-back signal, governing the direction of adaptation of the synapses during the learning procedure, is indispensable. Probably the simplest form of such a signal one can think of is a 'success' or 'failure' signal that is returned to the network after each attempt to associate the correct output to given input. On the basis of trial and error, a neural network can then indeed learn certain tasks, the principle on which 'reinforcement learning' is based [3,14]. This principle of reinforcement learning has a rather natural interpretation: satisfactory behavior is rewarded, or reinforced, causing this behavior to occur more frequently. The reinforcement signal is supplied by the subject's environment, or by its own judgment of the effect of its behavior. In a biological perspective, one could think of the synaptic change being influenced by some chemical substance, which is released depending on whether the evaluation by the subject of the effect of the output is positive or negative, i.e., whether it is happy or unhappy with the attempt it made.

Note that, in learning by reinforcement, the search for the correct output is more difficult, and, hence, slower, than for non-biologically realizable algorithms like the perceptron learning rule or back-propagation. This is not surprising, since the latter give the system locally specific information on how to adjust individual weights, while reinforcement rules only depend upon a global 'measure of correctness'.

The most general form of a reinforcement learning algorithm is given by the prescription

$$
\Delta w_{i j}=a_{i j}(r)+b_{i j}(r) x_{i}+c_{i j}(r) x_{j}+d_{i j}(r) x_{i} x_{j}
$$

Here the coefficients $a_{i j}, b_{i j}, c_{i j}, d_{i j}$, besides their dependence on the local variables such as the membrane potential, will in principle also depend on a reinforcement signal, denoted by $r$. The value of $r$ is usually a real number between 0 and 1 , denoting the degree of success $(r=1$ means success, $r=0$ means failure).

An important issue in the literature on reinforcement learning is the so called 'credit assignment problem' [14]. It refers to the question how a neural net knows which connections $w_{i j}$ were responsible for a successful or unsuccessful trial, and, as a consequence, which connections should be 'rewarded', and which should be 'punished', respectively.

In their article 'Learning from mistakes' (1999), Chialvo and Bak [4], proposed a class of networks, in which learning takes place on the basis of a 'deinforcement signal' only, i.e., the weights of active synapses are decreased if the output is wrong, they are 'punished', so to say, in case of wrong performance of the network. If the output is right nothing happens. This procedure 
works as long as the average activity in the net is kept very low: when only a few neurons are active at an unsuccessful attempt, one can be sure that the connections between these active neurons were the ones which were responsible, and thus should be 'punished'. In this way Chialvo and Bak obtained an elegant solution to the credit assignment problem.

The absence of a reinforcement signal (nothing happens if $r=1$ ) makes their learning rule relatively simple. It is a version of the general rule (5) with $b_{i j}=0$ and $c_{i j}=0$ :

$$
\Delta w_{i j}=-(1-r)\left(\rho x_{i} x_{j}-\varphi\right)
$$

where $\rho$ and $\varphi$ are positive constants; in this article we will suppose $\varphi<<\rho$. A biological mechanism that could implement the learning rule (6) is the following: if the output is correct, nothing happens, since the network obviously performs satisfactory. If not, a chemical substance is released, which has the effect that synapses between neurons that have just been active, and thereby are 'tagged' in some electro-chemical way, are depressed.

\subsection{Purpose}

The success of the 'minibrain model' of Chialvo and Bak [4] (as Wakeling and Bak referred to it in [15]), is limited to feed-forward neural nets in which the number of input and output neurons (or, equivalently in this model, the number of patterns) is small compared to the number of neurons in the hidden layer. As the number of neurons in the hidden layer decreases, learning, at a certain moment, becomes impossible: 'path interference' is the phenomenon which causes this effect [16]. Essentially, it amounts to the following. If, in each layer of the feed-forward neural net, only one neuron is active at each time step, an input-output relation corresponds to a path of activity along the strongest connections between the neurons. Basically, path interference comes down to the erasure of an existing path of activity, which was correct at a previous learning step, by a change due to a punishment of a connection while trying to learn a different input-output relation. If the probability for this path interference to occur becomes too large, learning times tend to infinity.

In this article we attempt to improve the performance of the minibrain model of Chialvo and Bak - in the sense of decreasing the learning time - by making sure that, at the occurrence of path interference, the punishment of a correct activity path is no longer such that the memory is erased. We achieve this by adding to the deinforcement term in the learning rule (6), which is proportional to $\rho$, a Hebbian term proportional to $\eta$. The latter term has the effect that an active path is strengthened, mitigating in this way the punishment. 
By choosing the ratio between the coefficients $\eta$ and $\rho$ of both terms in the learning rule appropriately, we are able to reduce the number of learning steps significantly, without making the model less realistic from a biological point of view. In fact, in the class of models we study, Hebbian learning is a most appropriate way to account for biological observations like LTP and LTD [8]. In section 4 we explain that if the quotient of the Hebbian learning rate and the coefficient of the deinforcement term is in the range

$$
\frac{1}{4}<\frac{\eta}{\rho}<\frac{1}{2}
$$

learning times are reduced considerably.

In their article [2], Chialvo and Bak proposed a different way to solve the problem of path interference. They reduced the amount of punishment of the connections that once had been active in a correct response. In this model a neuron needs to memorize whether it previously was active in a successful trial. In our model such a neuron memory is not needed.

Let us denote the deinforcement contribution to learning by $\Delta w_{i j}^{\prime}$ and denote the Hebbian part by $\Delta w_{i j}^{\prime \prime}$. We will study a learning rule of the form

$$
\Delta w_{i j}=\Delta w_{i j}^{\prime}+\Delta w_{i j}^{\prime \prime}
$$

From all possibilities for Hebbian learning summarized by equation (3), we choose for $\Delta w_{i j}^{\prime \prime}$ a rule in which the coefficients $a_{i j}$ and $b_{i j}$ both are zero:

$$
\Delta w_{i j}^{\prime \prime}=\varepsilon\left(x_{i}, x_{j}\right)\left(2 x_{i}-1\right) x_{j}
$$

We choose this particular form since it can be argued that this form is a most plausible candidate from a biological point of view [6].

Our paper has been set up as follows. In section 2, we describe a feed-forward network with one hidden layer, which we will use to study the learning rule (8), with (6) and (9). In section 3, numerical studies of various situations are given and explained. It turns out, in general, that taking into account Hebbian learning, and viewing it as a process which is permanently active, irrespective of the occurrence of reinforcement learning, has a positive influence on the learning time of the neural net. This is a new result, which, to the best of our knowledge, has not been noticed before. 


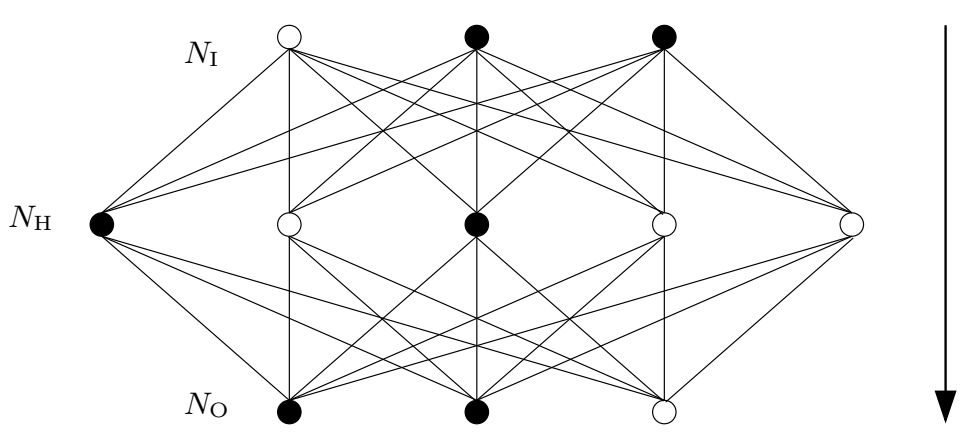

Fig. 1. An example of a fully connected feed forward network with $N_{\mathrm{I}}$ input neurons, $N_{\mathrm{H}}$ hidden neurons and $N_{\mathrm{O}}$ output neurons. The filled circles represent active neurons.

\section{Description of the model: updating rules for neuron activities and connection weights}

In order to explore a simplified model of the brain we consider a fully connected, feed-forward neural network with an input layer of $N_{\text {I neurons, one }}$ hidden layer of $N_{\mathrm{H}}$ neurons, and an output layer of $N_{\mathrm{O}}$ neurons, see figure 1 . The state $x_{i}$ of neuron $i$ is 1 if neuron $i$ fires and 0 if it does not. In general, a neuron fires if its potential $h_{i}$ is sufficiently high, where $h_{i}$ stands for the membrane potential $V_{\mathrm{ex}}-V_{\text {in }}$, the difference between the intra- and extra cellular potentials $V_{\text {in }}$ and $V_{\text {ex }}$. Following Chialvo and Bak [4], we model the dynamics of the neural net by simply stating that in the hidden and output layers a given number of neurons having the highest potentials $h_{i}$-in their respective layers - are those that will fire. In their terminology: we use extremal dynamics.

For McCulloch and Pitts neurons a way to control the average activity might be realized via suitably chosen threshold potentials $\vartheta_{i}$ (see e.g. [1], [3]). In nature, the average activity will depend on the threshold potentials and may, moreover, be influenced by chemical substances or the network structure $[8,10]$. In our model we put the threshold potentials $\theta_{i}$ equal to zero:

$$
\theta_{i}=0
$$

The number of neurons in the input, hidden and output layers that we choose to be active, will be denoted by $N_{\mathrm{I}}^{(\mathrm{a})}, N_{\mathrm{H}}^{(\mathrm{a})}$ and $N_{\mathrm{O}}^{(\mathrm{a})}$, respectively.

The input pattern, a specific set of states of neurons in the input layer, will be denoted by $\boldsymbol{\xi}_{\mathrm{I}}=\left(\xi_{\mathrm{I} 1}, \ldots, \xi_{\mathrm{I} N_{\mathrm{I}}}\right)$. The network is to associate every input pattern with a desired, or target, output pattern, $\boldsymbol{\xi}_{\mathrm{T}}=\left(\xi_{\mathrm{T} 1}, \ldots, \xi_{\mathrm{T} N_{\mathrm{O}}}\right)$. The $\boldsymbol{\xi}_{\mathrm{I}}$ and $\boldsymbol{\xi}_{\mathrm{T}}$ are vectors with components equal to 0 or 1 . Consequently, the number of active neurons of the input and output layer are given by 


$$
\begin{aligned}
& N_{\mathrm{I}}^{(\mathrm{a})}=\sum_{j=1}^{N_{\mathrm{I}}} \xi_{\mathrm{I} j} \\
& N_{\mathrm{O}}^{(\mathrm{a})}=\sum_{j=1}^{N_{\mathrm{O}}} \xi_{\mathrm{T} j}
\end{aligned}
$$

In our numerical experiments, these numbers will be taken to be equal. Moreover, the number of active neurons in the hidden layer,

$$
N_{\mathrm{H}}^{(\mathrm{a})}=\sum_{j=1}^{N_{\mathrm{H}}} x_{\mathrm{H} j}
$$

where $x_{\mathrm{H} j}$ is the neuron state of neuron $j$ in the hidden layer, will also be equal to the number of active neurons in the other layers. Hence, we choose $N_{\mathrm{I}}^{(\mathrm{a})}=N_{\mathrm{O}}^{(\mathrm{a})}=N_{\mathrm{H}}^{(\mathrm{a})}$.

We thus have explicitated the network dynamics. We now come to the update rules for the synaptic weights $w_{i j}$. Updating of the network state will happen at discrete time steps. At every time step $t_{n}$, all neuron states are updated in the order: input layer - hidden layer - output layer. This being done, the values of the weights are updated, according to

$$
w_{i j}\left(t_{n+1}\right)=w_{i j}\left(t_{n}\right)+\Delta w_{i j}\left(t_{n}\right)
$$

Substituting (9) and (6) into (8), we find

$$
\Delta w_{i j}=\varepsilon\left(x_{i}, x_{j}\right)\left(2 x_{i}-1\right) x_{j}-(1-r)\left(\rho x_{i} x_{j}-\varphi\right)
$$

For the pre-factor of the Hebbian term we take [6]

$$
\varepsilon\left(x_{i}, x_{j}\right)=\eta\left(\kappa-\left(2 x_{i}-1\right)\left(h_{i}-\theta_{i}\right)\right)
$$

The constants $\eta$ and $\kappa$ are positive numbers, the so-called learning rate and margin parameter. Finally, combining the above ingredients and noting that we chose $\theta_{i}=0$, the learning rule reads:

$$
\begin{aligned}
\Delta w_{i j}\left(t_{n}\right)= & \eta\left[\kappa-h_{i}\left(t_{n}\right)\left(2 x_{i}\left(t_{n}\right)-1\right)\right]\left[2 x_{i}\left(t_{n}\right)-1\right] x_{j}\left(t_{n}\right) \\
& +(1-r)\left[-\rho x_{i}\left(t_{n}\right) x_{j}\left(t_{n}\right)+\varphi\right]
\end{aligned}
$$

Note that $x_{i}\left(t_{n}\right)$ and $x_{j}\left(t_{n}\right)$ are the activities of neurons in adjacent layers, since in our model there are no lateral connections. The constant $\varphi$ is chosen in such a way that the change in $\sum_{i, j} w_{i j}$, where the sum is extended over $i$ and $j$ in adjacent layers, due to the $\rho$-term (not due to the Hebbian term), 
is independent of $\rho$. This can easily be achieved by choosing $\varphi=\rho / P$, where $P$ is the product of the numbers of neurons in two adjacent layers, i.e., $\varphi$ is equal to either $\rho / N_{\mathrm{I}} N_{\mathrm{H}}$ or $\rho / N_{\mathrm{H}} N_{\mathrm{O}}$.

\section{Numerical Simulations}

The network described in the previous section will now first be studied numerically. The numerical details are:

- The initial weights $w_{i j}\left(t_{0}\right)$ are randomly chosen with values between -0.01 and +0.01 .

- The punishment rate $\rho$ will be kept constant at 0.02 . Thus when we vary the $\eta / \rho$ ratio, we vary the learning rate $\eta$.

- The margin parameter $\kappa$, appearing in (16), will be kept at the value 1 .

- Whenever the number of neurons in the input, hidden or output layer is fixed, we choose $N_{\mathrm{I}}=8, N_{\mathrm{H}}=512$ and $N_{\mathrm{O}}=8$.

- All data are averaged over 512 samples.

The network is confronted with $p$ different input patterns $\boldsymbol{\xi}_{\mathrm{I}}^{\mu},(\mu=1, \ldots, p)$, to which correspond equally many target patterns $\boldsymbol{\xi}_{\mathrm{T}}^{\mu}$. Learning proceeds as follows. The input layer remains clamped to the first input pattern until the time step at which the target pattern has been found. As soon as this inputoutput relation $\mu=1$ has been learned, we switch to input pattern $\mu=2$. After the corresponding target pattern has been found we continue, up to the $p$-th input-output relation. Then, we have completed what we will refer to as one 'learning cycle'.

After this cycle we start the process again, up to the point where the network can recall all $p$ input-target relations at once. When that is the case, learning stops. We count the number of learning steps needed to learn all input-output relations.

Chialvo and Bak consider the case of one active neuron in each layer. In section 3.1 we present a numerical experiment with a neural network for which the activities are larger than one, i.e., $N_{\mathrm{I}}^{(\mathrm{a})}>1, N_{\mathrm{H}}^{(\mathrm{a})}>1$ and $N_{\mathrm{O}}^{(\mathrm{a})}>1$. In particular we study the total number of learning steps as a function of the ratio $\eta / \rho$. In section 3.2 we vary the number of neurons in input and output layer and keep the hidden layer fixed, and vice versa. Finally, in section 4, we interpret our results. 


\subsection{Effect of the Hebbian term}

In 'Learning from mistakes' Chialvo and Bak [4] studied the case of one active neuron in the input, the hidden and the output layers: $N_{\mathrm{I}}^{(\mathrm{a})}=N_{\mathrm{H}}^{(\mathrm{a})}=N_{\mathrm{O}}^{(\mathrm{a})}=1$. We here will study what happens when $N_{\mathrm{I}}^{(\mathrm{a})}, N_{\mathrm{H}}^{(\mathrm{a})}$ and $N_{\mathrm{O}}^{(\mathrm{a})}$ all are larger than one.

In our first numerical experiment we take a network with $p=8$ input-target relations for which, in each input or target pattern $\mu$, the number of active neurons is 2, i.e., $N_{\mathrm{I}}^{(\mathrm{a})}=N_{\mathrm{H}}^{(\mathrm{a})}=N_{\mathrm{O}}^{(\mathrm{a})}=2$. In figure 2 the number of learning steps is plotted against the ratio $\eta / \rho$ of the two proportionality coefficients related to the Hebbian and the deinforcement term respectively.

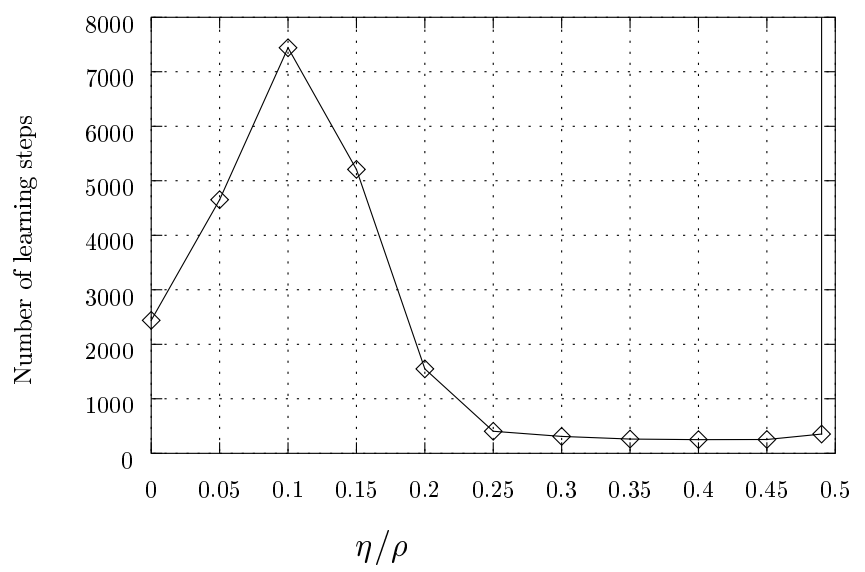

Fig. 2. The number of learning steps as a function of the quotient $\eta / \rho$. There are eight input-target relations to be learned and two active neurons in each layer. The number of neurons in the input, hidden and output layers are $N_{\mathrm{I}}=8, N_{\mathrm{H}}=512$ and $N_{\mathrm{O}}=8$. Initially, the number of learning steps increases as a result of the Hebbian learning term, but at $\eta / \rho=0.1$ the number of learning steps starts to decrease dramatically from 7500 to 250 at $\eta / \rho=0.25$. For $\eta / \rho>0.50$, learning is impossible.

From figure 2 we see that, when there is no Hebbian component in the learning rule $(\eta=0)$, the net needs 2500 learning steps to learn an input-output task. When we add a slight Hebbian component ( $\eta$ small) the number of learning steps increases, and, hence, the ability of the net to learn diminishes. However, when the Hebbian component becomes more and more important, the number of learning steps starts to decrease dramatically: for $\eta / \rho$ between 0.25 and 0.5 the number of learning steps is approximately 250. The Hebbian component, which has the tendency to engrave previously learned patterns, seems to help to not forget the old patterns. If $\eta / \rho$ exceeds the value 0.5 , learning fails. Apparently, the 'progressive' $\rho$ term, the power of which is to help the network to quickly adapt itself to new input-output relations, cannot conquer the 'conservative' power of the $\eta$-dependent (i.e., the Hebbian) term. 
We will come back to these points and consider the effects of the $\eta$ and $\rho$ terms in some detail in section 4 .

\subsection{Size dependences}

In this section we consider the network of figure 1 for varying numbers $N_{\mathrm{I}}=$ $N_{\mathrm{O}}$ and $N_{\mathrm{H}}$.

\subsubsection{Effect of varying the sizes of the input and output layers}

In this subsection we test the performance of the network for various sizes of the input, output and hidden layers. In particular, we chose to study the size-dependence for three different values of the learning parameter: $\eta / \rho=0$, $\eta / \rho=0.10$ and $\eta / \rho=0.45$, values which we selected on the basis of the results of the previous subsection.

First, we take a network with the fixed number of 512 neurons in the hidden layer, and only one active neuron per layer. The input and output layers will consist of increasing, equal numbers of neurons, starting with $N_{\mathrm{I}}=N_{\mathrm{O}}=4$. Moreover, we choose the number of input-output relations $p$ to be learned equal to the number of neurons in the input and output layers. The input and output layers will be enlarged in steps of 4 neurons, up to $N_{\mathrm{I}}=N_{\mathrm{O}}=28$ neurons.

In Figure 3 we give the number of learning steps per pattern for the above mentioned three values of $\eta / \rho$. The positive effect of the addition of a Hebbian term to the learning rule becomes more and more convincing with increasing number of input-output relations to be learned.

\subsubsection{Effect of varying the size of the hidden layer}

Next we consider a network with 8 input neurons, 8 output neurons and 8 subsequent patterns. The number of active neurons is 2 for all input and target patterns. We vary the number of neurons in the hidden layer.

In Figure 4 we have plotted the number of learning steps as a function of the number of neurons in the hidden layer for three values of the quotient $\eta / \rho$. Note that, in agreement with figure 2 the number of learning steps is the largest for $\eta / \rho=0.1$ (the symbols $\square$ in figure 4). A suitably chosen value for the coefficient $\eta$ of the Hebbian term makes it possible for the network to perform satisfactory with very small number of neurons in the hidden layer (the symbols $\bigcirc$ in the figure). 


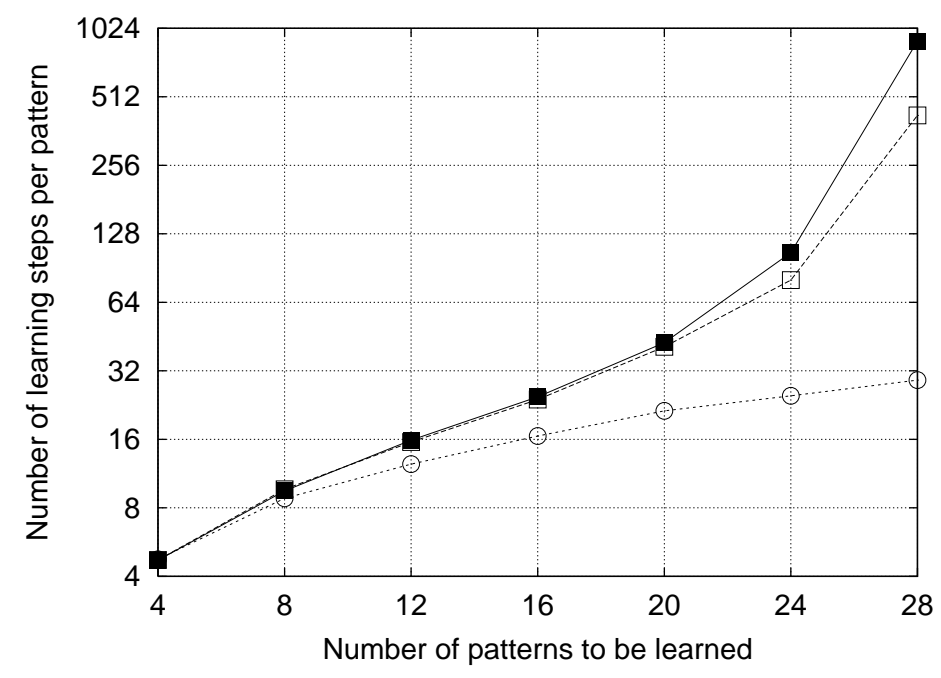

Fig. 3. The number of learning steps per pattern as a function of the number of input-output relations $p$, for $\eta / \rho=0(\boldsymbol{\square}), \eta / \rho=0.1(\square)$ and $\eta / \rho=0.45(\bigcirc)$. Input and output patterns have only one active neuron. The number of neurons in input and output layers equals the number of patterns $p$. Note the logarithmic scale of the vertical axis. For a small number of input-output patterns, the learning time is roughly equal for different values of $\eta / \rho$. The advantageous effect of a Hebbian term in the learning rule for this learning task becomes more and more pronounced with increasing numbers of input-target relations.

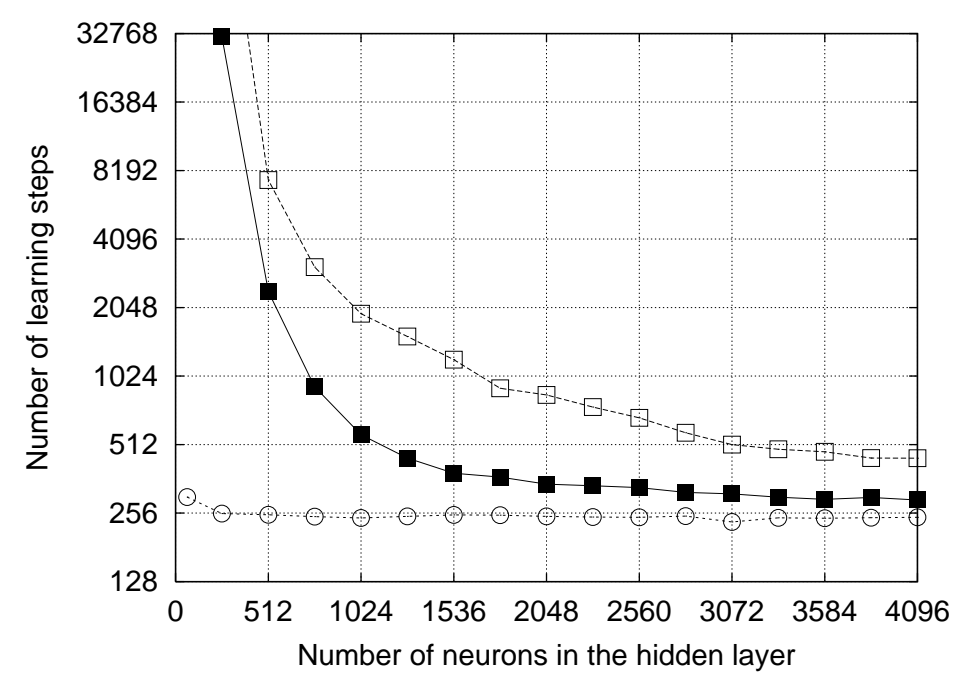

Fig. 4. Dependence of the number of learning steps on the number of neurons in the hidden layer of the network. The symbols $(\mathbf{\square}),(\square)$ and $(\bigcirc)$ correspond to $\eta / \rho=0$, $\eta / \rho=0.1$ and $\eta / \rho=0.45$ respectively. All input patterns and output patterns have 2 active neurons. The number of input neurons, output neurons and patterns are fixed; $N_{\mathrm{I}}=8, N_{\mathrm{O}}=8, p=8$. 


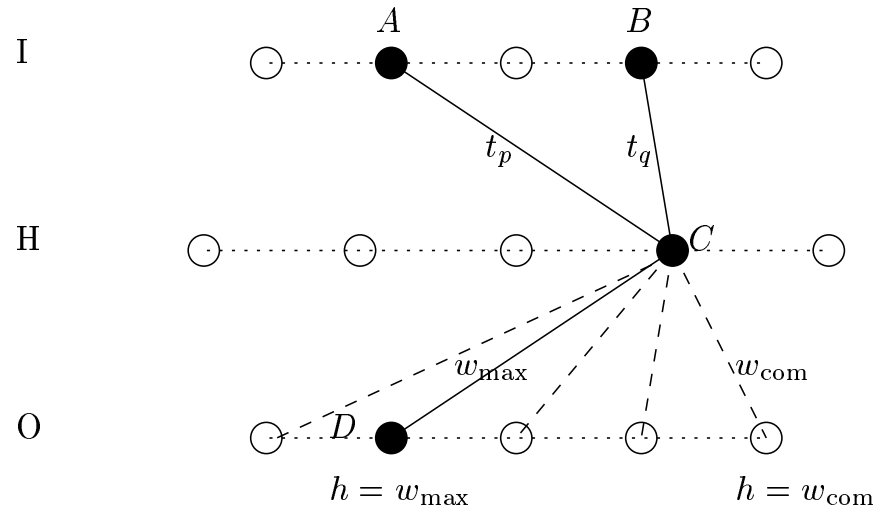

Fig. 5. Path interference At both the time steps $t_{p}$ and $t_{q}$ the neuron $C$ of the hidden layer fires, and as a result the same neuron $D$ of the output layer is activated. This unwanted effect is due to the fact that the connection of $B$ and $C$ happens to be the largest one. The paths $A C D$ and $B C D$ partially overlap.

\section{Explanation of the effect of the Hebbian term}

The different behavior for different values of $\eta / \rho$ is mainly due to its consequences for the effect we call path interference, after Wakeling [16], who studied the critical behavior of the Chialvo \& Bak minibrain.

As an example, let us consider the case in which only one neuron is active in each layer. In this case, the 'path of activity' from the active input neuron to the corresponding output neuron runs along the outgoing connections with the largest weights. During the learning process, it is possible that an established path (connecting, e.g., the active neuron of pattern $\boldsymbol{\xi}_{\text {I }}^{1}$ with the active neuron, in the output layer, of $\boldsymbol{\xi}_{\mathrm{O}}^{1}$ ) is 'wiped out' by an attempt to learn one of the other input-output relations. This is likely to happen if the same neuron in the hidden layer becomes active, and, consequently, the connection to the output neuron corresponding to the previously learned pattern is punished by an amount $\rho$. This phenomenon of path interference will occur once in a while, irrespective of the values of the parameters $\eta$ and $\rho$. However, the question whether the memory of the old pattern is wiped out (i.e., whether the connection to the output neuron under consideration is no longer the largest), does depend on the parameters $\rho$ and $\eta$. To find out how, we should consider the change of this connection compared to the change of the other connections from the same hidden neuron to the other output neurons. For the total relative change, two different learning steps should be taken into account. Firstly, the one at $t_{p}$, at which the right output was found, and the deinforcement term did not contribute, and, secondly, the learning step at $t_{q}$, at which path interference occurred, and the deinforcement term did contribute.

Let $w_{\text {win }}$ be the largest outgoing weight from the active neuron in the hidden 
layer to the output layer, and let $w_{\text {com }}$ be a weight value which is representative for one of the other, competing weights connecting the same neuron in the hidden layer to a different neuron in the output layer.

The membrane potentials of each neurons $i$ of the output layer are given, according to (4), by $h_{i}=w_{i j}$, where $j$ is a neuron of the hidden layer. From (17), with $x_{j}=1$, we find in case of success $(r=1)$ for the changes of the connections to the winning $\left(x_{i}\right)$ and the competing $\left(x_{i}=0\right)$ neurons in the output layer:

$$
\begin{aligned}
\Delta w_{\text {win }}\left(t_{p}\right) & =\eta\left[\kappa-w_{\text {win }}\left(t_{p}\right)\right] \\
\Delta w_{\text {com }}\left(t_{p}\right) & =-\eta\left(\kappa+w_{\text {com }}\left(t_{p}\right)\right)
\end{aligned}
$$

respectively. Similarly, in case of failure $(r=0)$ these changes are

$$
\begin{aligned}
\Delta w_{\text {win }}\left(t_{q}\right) & =\eta\left(\kappa-w_{\text {win }}\left(t_{q}\right)\right)-\rho+\varphi \\
\Delta w_{\text {com }}\left(t_{q}\right) & =-\eta\left(\kappa+w_{\text {com }}\left(t_{q}\right)\right)+\varphi
\end{aligned}
$$

respectively. Only if the increase of $w_{\text {com }}$ is larger than the increase of $w_{\text {win }}$, the memory path can be wiped out, since then $w_{\text {com }}$ may become the largest weight, i.e., if

$$
\Delta w_{\mathrm{com}}\left(t_{p}\right)+\Delta w_{\mathrm{com}}\left(t_{q}\right)>\Delta w_{\mathrm{win}}\left(t_{p}\right)+\Delta w_{\mathrm{win}}\left(t_{q}\right)
$$

We now substitute (18)-(21) into (22). In the resulting inequality we can ignore the values of $w_{\text {win }}$ and $w_{\text {com }}$ relative to $\kappa$ as long as the number of adaptations of $w_{\text {win }}$ and $w_{\text {com }}$ is small; recall that $\kappa=1, \rho=0.02$, and the initial values of the weights are in the range $[-0.01,0.01]$ in our numerical experiments. With these approximations, the inequality reduces to $\rho>4 \eta$. In the opposite case,

$$
\rho<4 \eta
$$

$w_{\text {win }}$ will remain larger than $w_{\text {com }}$ and, consequently, path interference will not wipe out learned input-output relations, which explains the decrease of the number of learning steps for $\eta>\frac{1}{4} \rho$. For $\eta>\frac{1}{2}$ the network is incapable of learning input-output relations. This can be seen as follows. Each time a winning connection is punished, i.e., the output is wrong, it changes approximately by an amount $\eta-\rho$, whereas the competing connection changes by an amount of $-\eta$. Hence, only when $\eta-\rho<-\eta$, or, equivalently, when

$$
2 \eta<\rho
$$

the winning connection decreases more than the competing connection. In 
the opposite case, $2 \eta>\rho$, the winning connection remains larger than its competitor, and, at the next learning step, the output will be wrong again.

Combining the inequalities (23) and (24), we find the central result of this article (7), the parameter region for which a Hebbian term in the learning rule is advantageous. This observation is confirmed by the numerical experiment of section 3.1, so, in particular, figure 2 .

Note that the reasoning leading to the main results (23) and (24) was based on an assumption regarding the initial values. In particular, it was assumed that the weights were small compared to $\kappa$ (which was put equal to 1 ). In reference [6] it was shown that the pre-factor (16) of the Hebbian term tends to zero during the learning process:

$$
\kappa-\left(2 x_{i}-1\right)\left(h_{i}-\theta_{i}\right) \rightarrow 0
$$

implying, that for a small number of active neurons the absolute values of the weights are of the order $\kappa$, as follows with (4) and (10). Hence, the assumption that the weights are small compared to $\kappa(\kappa=1)$ is guaranteed to break down at a certain point in the learning process.

\section{Summary}

We have shown, in a particular model, that a Hebbian component in a reinforcement rule improves the ability to learn input-output relations by a neural net.

\section{References}

[1] Alstrom P and Stassinopoulos D 1995 Phys. Rev. E 515027

[2] Bak P and Chialvo D R 2001 Phys. Rev. E 63031912

[3] Barto A G 1995 Reinforcement learning: The Handbook of Brain Theory and Neural Networks ed M A Arbib (Cambridge, Massachusetts: MIT Press) 804809

[4] Chialvo D R and Bak P 1999 Neuroscience 901137

[5] Hebb D O 1949 The Organization of Behaviour (New York: Wiley) 62

[6] Heerema M and van Leeuwen W A 1999 J. Phys. A: Math. Gen. 32263 
[7] Hertz J, Krogh A and Palmer R G 1991 Introduction to the theory of neural computation (Redwood City, California: Addison-Wesley)

[8] Kandel E R, Schwartz J H and Jessell T M 1991 Principles of Neural Science, Third Edition (Englewood-Cliffs, New Jersey: Prentice-Hall International Inc.)

[9] Klemm K, Bornhodt S and Schuster H G 2000 Phys. Rev. Lett. 841813

[10] Kolb B and Whishaw I Q 1990 Human Neuropsychology, 3rd edition (New York: Freeman)

[11] Minsky M L and Papert S A 1969 Perceptrons: An Introduction to Computational Geometry (Cambridge, Massachusetts: MIT Press)

[12] Müller B, Reinhardt J and Strickland M T 1990 Neural Networks, An Introduction (Berlin: Springer-Verlag)

[13] Rosenblatt F 1962 Principles of Neurodynamics (New York: Spartan)

[14] Sutton R S and Barto A G 1998 Reinforcement learning: An introduction (Cambridge, Massachusetts: MIT Press)

[15] Wakeling J and Bak P 2001 arXiv:nlin.AO/0201046

[16] Wakeling J 2002 cond-mat/0204562 